\title{
ON THE TYPE OF TRIANGLE GROUPS
}

\author{
Carlos H. Grossi \\ AbStract. We prove a conjecture of R. Schwartz about the type of some complex hyperbolic triangle groups.
}

\section{Introduction}

An $\left(n_{1}, n_{2}, n_{3}\right)$-complex reflection complex hyperbolic triangle group is a group of isometries of the complex hyperbolic plane $\mathbb{H}_{\mathbb{C}}^{2}$ generated by complex reflections $I_{1}, I_{2}, I_{3}$ in complex geodesics $C_{1}, C_{2}, C_{3}$ such that $C_{i}$ and $C_{i+1}$ meet at the angle $\pi / n_{i}, n_{i} \geq 2$ (see Section 2 for definitions). For fixed $n_{1}, n_{2}, n_{3}$, modulo conjugacy in $\operatorname{Isom}\left(\mathbb{H}_{\mathbb{C}}^{2}\right)$, there exists in general a 1-parameter family of $\left(n_{1}, n_{2}, n_{3}\right)$-triangle groups. Assume $n_{1} \leq n_{2} \leq n_{3}, n_{i} \in \mathbb{N}$. The triple $\left(n_{1}, n_{2}, n_{3}\right)$ is classified with respect to the behavior of the isometries

$$
W_{A}:=I_{3} I_{2} I_{1} I_{2} \quad \text { and } \quad W_{B}:=I_{1} I_{2} I_{3}
$$

while a parameter of the $\left(n_{1}, n_{2}, n_{3}\right)$-triangle group family varies in a canonical way. The triple is said to be of type $A$ if $W_{A}$ becomes regular elliptic before $W_{B}$ and of type $B$ if $W_{B}$ becomes regular elliptic before $W_{A}$ (see Subsections 2.2 and 2.3).

In this paper, we prove the following

1.1. Conjecture $\left[\mathrm{Sch} 2\right.$, Conjecture 5.2]. The triple $\left(n_{1}, n_{2}, n_{3}\right)$ has type $A$ if $n_{1} \leq 9$ and type $B$ if $n_{1} \geq 14$.

Conjecture 1.1 is a tiny portion of a complete conjectural picture [Sch2] that describes $\left(n_{1}, n_{2}, n_{3}\right)$ triangle groups with focus on discreteness. Roughly speaking, the picture is as follows: An $\left(n_{1}, n_{2}, n_{3}\right)$ triangle group is discrete if neither $W_{A}$ nor $W_{B}$ is regular elliptic. When $\left(n_{1}, n_{2}, n_{3}\right)$ has type $B$, then the converse also holds. If $\left(n_{1}, n_{2}, n_{3}\right)$ has type $A$, then there is a countable collection of 'extra' discrete groups. While sorting triangle groups into types $A$ and $B$ is (as we shall see) a matter of a couple of simple tricks intended to avoid the huge amount of calculus a straightforward approach leads to, classifying those groups with respect to discreteness is certainly a much more difficult and interesting task. It has been accomplished for $(\infty, \infty, \infty)$-triangle groups in [GoP] and [Sch1] (see also [Sch3]) and for $\left(n_{1}, n_{2}, n_{3}\right)$-triangle groups with sufficiently large $n_{1}$ in [Sch4].

In principle, we could say that our proof is computer independent. In fact, we use the computer only to obtain approximate values of the cosine function needed to establish some inequalities that hold by a wide margin of error (Lemmas 3.1 and 3.2). The inequalities proved in this way are marked with the symbols $\prec$ and $\succ$.

Conjecture 1.1 has been solved for sufficiently large $n_{1}$ in [Sch4] and for triples of the form $(n, n, \infty)$ in [W-G]. In [Pra], it is shown that the triples corresponding to triangles with $r_{1}^{2}+r_{2}^{2}+r_{3}^{2}-1=2 r_{1} r_{2} r_{3}$ and $r_{1} r_{2} r_{3} \geq \frac{13+\sqrt{297}}{32}$ (see [Pra] and also Subsection 2.2) are of type $B$.

Supported by FAPEMIG. 
Acknowledgments. The author is grateful to Sasha Anan'in and to Nikolay Gusevskii for many stimulating and fruitful discussions about complex hyperbolic geometry.

\section{Preliminaries}

2.1. Basic background. Let $V$ be a 3 -dimensional $\mathbb{C}$-vector space equipped with a hermitian form $\langle-,-\rangle$ of signature,,++- . The complex hyperbolic plane $\mathbb{H}_{\mathbb{C}}^{2}$ can be identified with the open 4 -ball ${ }^{1}$

$$
\text { B } V:=\left\{p \in \mathbb{P}_{\mathbb{C}} V \mid\langle p, p\rangle<0\right\} .
$$

The complex hyperbolic distance $d\left(p_{1}, p_{2}\right)$ between two points $p_{1}, p_{2} \in \mathrm{B} V$ is given in terms of the tance $^{2}$

$$
\operatorname{ta}\left(p_{1}, p_{2}\right):=\frac{\left\langle p_{1}, p_{2}\right\rangle\left\langle p_{2}, p_{1}\right\rangle}{\left\langle p_{1}, p_{1}\right\rangle\left\langle p_{2}, p_{2}\right\rangle}
$$

by $\cosh ^{2}\left(d\left(p_{1}, p_{2}\right) / 2\right)=\operatorname{ta}\left(p_{1}, p_{2}\right)$ [Gol, p. 77]. The ideal boundary of $\mathbb{H}_{\mathbb{C}}^{2}$ is the 3 -sphere

$$
\mathrm{S} V:=\left\{p \in \mathbb{P}_{\mathbb{C}} V \mid\langle p, p\rangle=0\right\}
$$

formed by the isotropic points in $\mathbb{P}_{\mathbb{C}} V$. Notice that the tance $\operatorname{ta}\left(p_{1}, p_{2}\right)$ is well-defined for all nonisotropic $p_{1}, p_{2} \in \mathbb{P}_{\mathbb{C}} V$. We put $\overline{\mathrm{B}} V:=\mathrm{B} V \cup \mathrm{S} V$.

Every projective line $L$ in $\mathbb{P}_{\mathbb{C}} V$ has the form $L=\mathbb{P}_{\mathbb{C}} p^{\perp}$, where $p \in \mathbb{P}_{\mathbb{C}} V$ and $p^{\perp}=\{v \in V \mid\langle v, p\rangle=0\}$. We call $p$ the polar point to $L$. If $p \notin \overline{\mathrm{B}} V$, then $\mathbb{P}_{\mathbb{C}} p^{\perp} \cap \overline{\mathrm{B}} V$ is a complex geodesic. Two distinct complex geodesics $C_{1}, C_{2}$ are concurrent (respectively, asymptotic, ultraparallel) if and only if $C_{1} \cap C_{2} \in \mathrm{B} V$ (respectively, $C_{1} \cap C_{2} \in \mathrm{S} V, C_{1} \cap C_{2}=\varnothing$ ).

2.1.1. Lemma [Gol, p. 100]. Two distinct complex geodesics $C_{1}, C_{2}$ with polar points $p_{1}, p_{2}$ are concurrent, asymptotic, ultraparallel if and only if $\operatorname{ta}\left(p_{1}, p_{2}\right)<1, \operatorname{ta}\left(p_{1}, p_{2}\right)=1, \operatorname{ta}\left(p_{1}, p_{2}\right)>1$, respectively. If $\mathrm{ta}\left(p_{1}, p_{2}\right) \leq 1$, then the angle $\angle\left(C_{1}, C_{2}\right) \in[0, \pi / 2]$ between $C_{1}$ and $C_{2}$ is given by $\cos ^{2} \angle\left(C_{1}, C_{2}\right)=\operatorname{ta}\left(p_{1}, p_{2}\right)$

Given $p \notin \overline{\mathrm{B}} V$, define $I \in \mathrm{SU} V$ by the rule

$$
I: x \mapsto 2 \frac{\langle x, p\rangle}{\langle p, p\rangle} p-x .
$$

The corresponding isometry in $\mathrm{PU} V$ is known as the complex reflection in the complex geodesic $\mathbb{P}_{\mathbb{C}} p^{\perp} \cap$ $\overline{\mathrm{B}} V$. For brevity, we will call it simply the reflection in the complex geodesic in question.

2.2. Complex hyperbolic triangles. A complex hyperbolic triangle is a triple $\left(C_{1}, C_{2}, C_{3}\right)$ of complex geodesics in $\overline{\mathrm{B}} V$. Each complex geodesic $C_{i}$ is a side of the triangle. If the sides $C_{i}$ and $C_{i+1}$ meet at the angle $\pi / n_{i}$, where $n_{i} \geq 2$ (we allow $n_{i}$ to be infinite, meaning that $C_{i}$ and $C_{i+1}$ are asymptotic or equal), the triangle $\left(C_{1}, C_{2}, C_{3}\right)$ is referred to as an $\left(n_{1}, n_{2}, n_{3}\right)$-triangle. We call an $\left(n_{1}, n_{2}, n_{3}\right)$-triangle $\left(C_{1}, C_{2}, C_{3}\right)$ nondegenerate if the form restricted to the subspace $\mathbb{C} p_{1}+\mathbb{C} p_{2}+\mathbb{C} p_{3}$ of $V$ is nondegenerate, being $p_{i}$ the polar point to $C_{i}$.

2.2.1. Lemma (compare with [Pra, Proposition 1]). Let $\left(C_{1}, C_{2}, C_{3}\right)$ be a nondegenerate $\left(n_{1}, n_{2}, n_{3}\right)$ triangle with $n_{i}>2, i=1,2,3$. Denote by $p_{i}$ the polar point to $C_{i}$. Define

$$
r_{i}:=\sqrt{\operatorname{ta}\left(p_{i}, p_{i+1}\right)}=\cos \frac{\pi}{n_{i}}>0, \quad \varkappa:=\frac{\left\langle p_{1}, p_{2}\right\rangle\left\langle p_{2}, p_{3}\right\rangle\left\langle p_{3}, p_{1}\right\rangle}{\left\langle p_{1}, p_{1}\right\rangle\left\langle p_{2}, p_{2}\right\rangle\left\langle p_{3}, p_{3}\right\rangle}, \quad \varepsilon:=\frac{\varkappa}{|\varkappa|}, \quad t:=\operatorname{Re} \varepsilon .
$$

\footnotetext{
${ }^{1}$ The symbol $:=$ stands for 'equals by definition.'

${ }^{2}$ Here, and in what follows, we frequently do not distinguish the notation of a point in $\mathbb{P}_{\mathbb{C}} V$ and of a chosen representative of it in $V$ when a concept or expression does not depend on such a choice.
} 
Then, the numbers $r_{i}$ and $t$ constitute a complete set of geometrical invariants of $\left(C_{1}, C_{2}, C_{3}\right)$. They satisfy $0<r_{i} \leq 1,|t| \leq 1$, and

$$
1+2 r_{1} r_{2} r_{3} t-\left(r_{1}^{2}+r_{2}^{2}+r_{3}^{2}\right) \leq 0 .
$$

All values of $r_{i}$ and $t$ subject to the conditions $0<r_{i} \leq 1,|t| \leq 1$, and $1+2 r_{1} r_{2} r_{3} t-\left(r_{1}^{2}+r_{2}^{2}+r_{3}^{2}\right) \leq 0$ correspond to a nondegenerate $\left(n_{1}, n_{2}, n_{3}\right)$-triangle with $n_{i}>2$.

Proof. The numbers $r_{i}$ and $\varepsilon$ are invariant under the action of $\mathrm{PU} V$ on the triple $\left(p_{1}, p_{2}, p_{3}\right)$. Choosing suitable representatives $p_{i} \in V$, we can assume that

$$
\left(\begin{array}{ccc}
1 & r_{1} & r_{3} \bar{\varepsilon} \\
r_{1} & 1 & r_{2} \\
r_{3} \varepsilon & r_{2} & 1
\end{array}\right)
$$

is the Gram matrix of $\left(p_{1}, p_{2}, p_{3}\right)$. If the triples $\left(p_{1}, p_{2}, p_{3}\right)$ and $\left(p_{1}^{\prime}, p_{2}^{\prime}, p_{3}^{\prime}\right)$ have the same Gram matrix and if the hermitian form is nondegenerate being restricted to the subspaces generated by $p_{1}, p_{2}, p_{3}$ and by $p_{1}^{\prime}, p_{2}^{\prime}, p_{3}^{\prime}$, then there exists $I \in \mathrm{U} V$ such that $I p_{i}=p_{i}^{\prime}$. The triangles corresponding to $\left(r_{1}, r_{2}, r_{3}, \varepsilon\right)$ and to $\left(r_{1}^{\prime}, r_{2}^{\prime}, r_{3}^{\prime}, \varepsilon^{\prime}\right)$ differ by an anti-holomorphic isometry of $\mathbb{H}_{\mathbb{C}}^{2}$ if and only if $r_{i}=r_{i}^{\prime}$ and $\varepsilon=\bar{\varepsilon}^{\prime}$. The rest follows from Sylvester's criterion

From now on, all $\left(n_{1}, n_{2}, n_{3}\right)$-triangles are assumed to be nondegenerate.

2.2.4. Remark. For fixed $3<n_{1} \leq n_{2} \leq n_{3}$, there exists a non-empty 1-parameter family of $\left(n_{1}, n_{2}, n_{3}\right)$-triangles. Indeed, the left-hand side of the inequality (2.2.2) is increasing in $t$ and, hence, attains its minimum value at $t=-1$. We have

$$
1-2 r_{1} r_{2} r_{3}-\left(r_{1}^{2}+r_{2}^{2}+r_{3}^{2}\right)<1-2 \cos ^{3} \frac{\pi}{3}-3 \cos ^{2} \frac{\pi}{3}=0
$$

Let $3<n_{1} \leq n_{2} \leq n_{3}$. In the terms of Lemma 2.2.1, define

$$
t_{M}:=\frac{r_{1}^{2}+r_{2}^{2}+r_{3}^{2}-1}{2 r_{1} r_{2} r_{3}}, \quad t_{\max }:=\min \left\{t_{M}, 1\right\} .
$$

The canonical path of deformation of the $\left(n_{1}, n_{2}, n_{3}\right)$-triangle family is the one that starts with $t=-1$ and ends with $t=t_{\max }$.

2.3. Complex hyperbolic triangle groups. The subgroup in PU $V$ generated by the reflections in the sides of an $\left(n_{1}, n_{2}, n_{3}\right)$-triangle is called an $\left(n_{1}, n_{2}, n_{3}\right)$-triangle group. Up to conjugacy in $\operatorname{Isom}\left(\mathbb{H}_{\mathbb{C}}^{2}\right)$, all nondegenerate $\left(n_{1}, n_{2}, n_{3}\right)$-triangle groups are described by Lemma 2.2.1.

Fix $3<n_{1} \leq n_{2} \leq n_{3}$ and assume $n_{i} \in \mathbb{N}$. For a given $\left(n_{1}, n_{2}, n_{3}\right)$-triangle $\left(C_{1}, C_{2}, C_{3}\right)$, define

$$
W_{A}:=I_{3} I_{2} I_{1} I_{2}, \quad W_{B}:=I_{1} I_{2} I_{3},
$$

where $I_{i}$ stands for the reflection in $C_{i}$. The triple $\left(n_{1}, n_{2}, n_{3}\right)$ is characterized with respect to the behavior of the isometries $W_{A}$ and $W_{B}$ during the canonical deformation of the 1-parameter family of $\left(n_{1}, n_{2}, n_{3}\right)$-triangle groups. Specifically, $\left(n_{1}, n_{2}, n_{3}\right)$ is said to be of type $A$ if $W_{A}$ becomes regular elliptic before $W_{B}$ and of type $B$ if $W_{B}$ becomes regular elliptic before $W_{A}$ (see [Gol] for the classification of holomorphic isometries of $\left.\mathbb{H}_{\mathbb{C}}^{2}\right)$.

2.3.1. Lemma (compare with [Pra, Proposition 12]). $W_{A}$ is always hyperbolic at the beginning of the deformation. In the terms of Lemma 2.2.1, $W_{A}$ is regular elliptic if and only if

$$
t>t_{W_{A}}:=\frac{r_{3}^{2}+4 r_{1}^{2} r_{2}^{2}-1}{4 r_{1} r_{2} r_{3}} .
$$


Proof. Let $\left(C_{1}, C_{2}, C_{3}\right)$ be an $\left(n_{1}, n_{2}, n_{3}\right)$-triangle and let $p_{i}$ denote the polar point to $C_{i}$. The isometry $W_{A}$ is the product of two reflections: one in the complex geodesic with polar point $I_{2} p_{1}$ and the other in $C_{3}$. The nature of $W_{A}$ is hence determined by the relative position of these complex geodesics. Taking (2.2.3) as the Gram matrix of suitable representatives $p_{i} \in V$ and applying (2.1.2), we obtain

$$
\operatorname{ta}\left(I_{2} p_{1}, p_{3}\right)=\frac{\left\langle I_{2} p_{1}, p_{3}\right\rangle\left\langle p_{3}, I_{2} p_{1}\right\rangle}{\left\langle I_{2} p_{1}, I_{2} p_{1}\right\rangle\left\langle p_{3}, p_{3}\right\rangle}=\left|\left\langle 2 r_{1} p_{2}-p_{1}, p_{3}\right\rangle\right|^{2}=4 r_{1}^{2} r_{2}^{2}-4 r_{1} r_{2} r_{3} t+r_{3}^{2} .
$$

At the beginning of the deformation,

$$
\operatorname{ta}\left(I_{2} p_{1}, p_{3}\right)=4 r_{1}^{2} r_{2}^{2}+4 r_{1} r_{2} r_{3}+r_{3}^{2}>4\left(\cos ^{4} \frac{\pi}{3}+\cos ^{3} \frac{\pi}{3}\right)+\cos ^{2} \frac{\pi}{3}=1 .
$$

By Lemma 2.1.1, this implies that $W_{A}$ is hyperbolic. It remains to observe that $\operatorname{ta}\left(I_{2} p_{1}, p_{3}\right)$ is decreasing in $t$ and that, by Lemma 2.1.1, $W_{A}$ becomes parabolic exactly when $t=t_{W_{A}}$

In order to deal with $W_{B}$, we need the following

2.3.2. Lemma [Gol, Theorem 6.2.4]. Define a map $f: \mathbb{C} \rightarrow \mathbb{R}$ by

$$
f(z):=|z|^{4}-8 \operatorname{Re}\left(z^{3}\right)+18|z|^{2}-27 .
$$

Given $J \in \mathrm{PU} V$, let $\hat{J} \in \mathrm{SU} V$ be a lift of $J$. Then, $J$ is regular elliptic (respectively, loxodromic) if and only if $f(\operatorname{tr} \hat{J})<0$ (respectively, $f(\operatorname{tr} \hat{J})>0$ ). The pre-image $f^{-1}(0) \subset \mathbb{C}$ is a deltoid. If $\operatorname{tr} \hat{J} \in \mathbb{R}$, then $J$ is loxodromic if and only if $\operatorname{tr} \hat{J} \notin[-1,3]$ by

Take the lift $W_{B} \in \mathrm{SU} V$ determined by the lifts of $I_{1}, I_{2}, I_{3}$ in (2.1.2). The trace $\tau:=\operatorname{tr} W_{B}$ is given

$$
\tau:=\operatorname{tr} W_{B}=8 r_{1} r_{2} r_{3} \varepsilon-4\left(r_{1}^{2}+r_{2}^{2}+r_{3}^{2}\right)+3 \in \mathbb{C}
$$

(see, for instance, [Pra]).

2.3.4. Lemma. $W_{B}$ is always loxodromic at the beginning of the deformation.

Proof. By (2.3.3),

$$
\tau=-8 r_{1} r_{2} r_{3}-4\left(r_{1}^{2}+r_{2}^{2}+r_{3}^{2}\right)+3<-8 \cos ^{3} \frac{\pi}{3}-12 \cos ^{2} \frac{\pi}{3}+3=-1
$$

at the beginning of the deformation. The result follows from Lemma 2.3.2

During the deformation, $\tau$ belongs to the circle

$$
F:=\left\{(x, y) \in \mathbb{C} \mid\left(x+4\left(r_{1}^{2}+r_{2}^{2}+r_{3}^{2}\right)-3\right)^{2}+y^{2}=\left(8 r_{1} r_{2} r_{3}\right)^{2}\right\},
$$

where $x:=\operatorname{Re} \tau$ and $y:=\operatorname{Im} \tau$. By Lemma 2.2.1, we can assume that $\operatorname{Im} \varepsilon \geq 0$, i.e., $y \geq 0$. By (2.3.3), the coordinate $x=\operatorname{Re} \tau$ and the parameter $t=\operatorname{Re} \varepsilon$ are linked by the formula

$$
t=\frac{x+4\left(r_{1}^{2}+r_{2}^{2}+r_{3}^{2}\right)-3}{8 r_{1} r_{2} r_{3}}
$$

Hence, we can think of the parameter $t$ as 'living' in the upper half-circle of $F$. 


\section{Proof of the conjecture}

In what follows, we will refer to the elementary Lemmas 4.1 and 4.3, proved in Section 4.

3.1. Proposition. If $14 \leq n_{1} \leq n_{2} \leq n_{3}$, then the triple $\left(n_{1}, n_{2}, n_{3}\right)$ is of type $B$.

Proof. In the terms of Lemma 2.2.1, $\cos \frac{\pi}{14} \leq r_{1} \leq r_{2} \leq r_{3} \leq 1$.

Intersection points $(x,-\sqrt{3} x) \in \mathbb{C}$ of the line $y=-\sqrt{3} x$ that passes through the vertex $\left(-\frac{3}{2}, \frac{3 \sqrt{3}}{2}\right)$ and through the center $(0,0)$ of Goldman's deltoid (Lemma 2.3.2) with the circle $F$ given by (2.3.5) satisfy $^{3}$

$$
4 x^{2}+2\left(4 \sum r_{i}^{2}-3\right) x+\left(4 \sum r_{i}^{2}-3\right)^{2}-\left(8 r_{1} r_{2} r_{3}\right)^{2}=0 .
$$

By Lemma 4.1 (1), the discriminant

$$
D_{1}:=4\left(-3\left(4 \sum r_{i}^{2}-3\right)^{2}+\left(16 r_{1} r_{2} r_{3}\right)^{2}\right)
$$

of the above equation is such that $D_{1}>0$. Take the root $x_{0}:=\frac{-2\left(4 \sum r_{i}^{2}-3\right)+\sqrt{D_{1}}}{8}$.

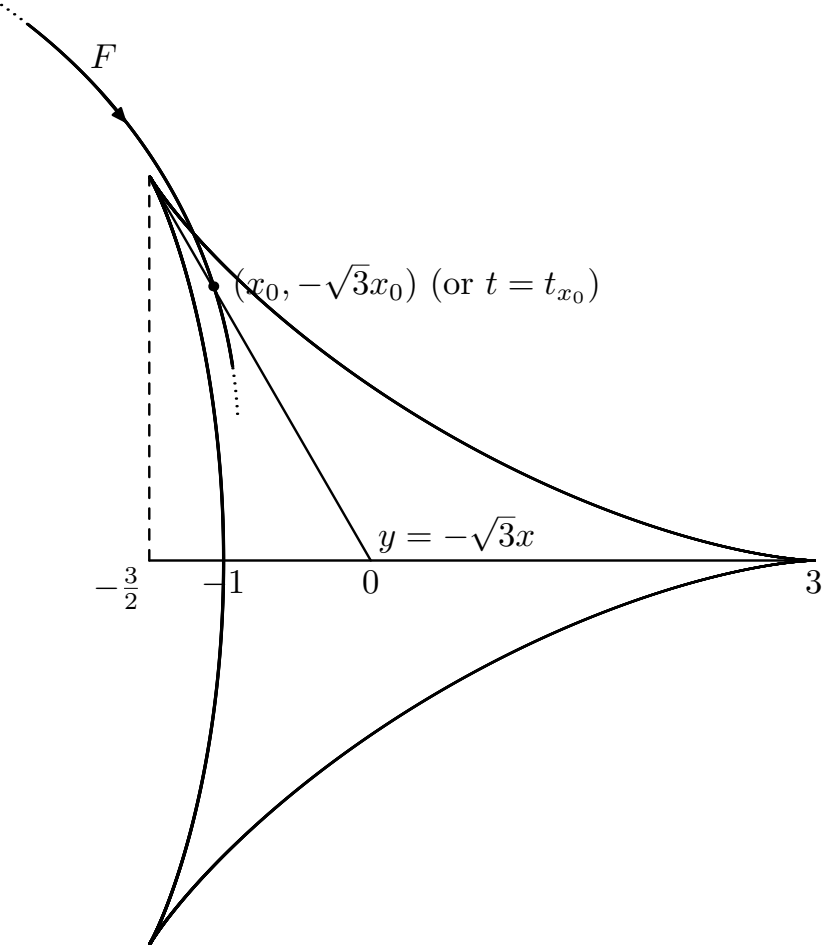

We will show that $x_{0} \in\left(-\frac{3}{2},-1\right)$. In particular, this implies that $\left(x_{0},-\sqrt{3} x_{0}\right) \in F$ is 'inside of' Goldman's deltoid. ${ }^{4}$ The inequality $x_{0}<-1$, equivalent to $\sqrt{D_{1}}<2\left(4 \sum r_{i}^{2}-3\right)-8$, follows from Lemma 4.1 (2) and from ${ }^{5}$

$$
2\left(4 \sum r_{i}^{2}-3\right)-8 \geq 2\left(12 \cos ^{2} \frac{\pi}{14}-3\right)-8 \succ 8.8 .
$$

\footnotetext{
${ }^{3}$ Obviously, we always sum over $i=1,2,3$.

${ }^{4}$ It would suffice to prove here a weaker inequality, but we will need later the fact that $x_{0}<-1$.

${ }^{5}$ As stated in the introduction, the symbols $\prec$ and $\succ$ are used for the inequalities proved using the computer to find approximate values of the cosine function.
} 
The inequality $x_{0}>-\frac{3}{2}$ follows from $-\left(4 \sum r_{i}^{2}-3\right)^{2}+3\left(4 \sum r_{i}^{2}-3\right)+\left(8 r_{1} r_{2} r_{3}\right)^{2}-9>0$ which is a consequence of Lemma 4.1 (3).

According to (2.3.6), the value of the deformation parameter $t$ that corresponds to $x_{0}$ is

$$
t_{x_{0}}:=\frac{x_{0}+4 \sum_{i} r_{i}^{2}-3}{8 r_{1} r_{2} r_{3}}
$$

It satisfies $t_{x_{0}} \in\left(-1, t_{\max }\right)$, being $t_{\max }$ as defined in (2.2.5). Indeed, the inequality $t_{x_{0}}>-1$ is straightforward. The inequality $t_{x_{0}}<1$, equivalent to $\sqrt{D_{1}}<-6\left(4 \sum r_{i}^{2}-3\right)+64 r_{1} r_{2} r_{3}$, follows from Lemma 4.1 (2), (4). Finally, the inequality $t_{x_{0}}<t_{M}$ is equivalent to $x_{0}<-1$.

We have just proved that the deformation parameter assumes the value $t=t_{x_{0}}$. By Lemma 2.3.2, the isometry $W_{B}$ is regular elliptic when $t=t_{x_{0}}$ since $\left(x_{0},-\sqrt{3} x_{0}\right)$ is inside of the deltoid. By Lemma 2.3.1, in order to show that $W_{B}$ becomes regular elliptic before $W_{A}$, it suffices to show that $t_{x_{0}}<t_{W_{A}}$. This follows from

$$
3\left(4 \sum r_{i}^{2}-3\right)^{2}-12\left(r_{3}^{2}+4 r_{1}^{2} r_{2}^{2}-1\right)\left(4 \sum r_{i}^{2}-3\right)+16\left(r_{3}^{2}+4 r_{1}^{2} r_{2}^{2}-1\right)^{2}-\left(8 r_{1} r_{2} r_{3}\right)^{2}>0
$$

which is a consequence of Lemma $4.1(5)$

3.5. Proposition. If $n_{1} \leq n_{2} \leq n_{3}$ and $4 \leq n_{1} \leq 9$, then the triple $\left(n_{1}, n_{2}, n_{3}\right)$ is of type $A$.

Proof. In the terms of Lemma 2.2.1, $r_{1} \leq r_{2} \leq r_{3} \leq 1$ and $\cos \frac{\pi}{4} \leq r_{1} \leq \cos \frac{\pi}{9}$.

First, let us show that the deformation parameter $t$ assumes the value $t=t_{W_{A}}$, being $t_{W_{A}}$ as in Lemma 2.3.1. In other words, we need to show that $t_{W_{A}} \in\left[-1, t_{\max }\right]$ (see 2.2.5). The inequalities $t_{W_{A}}>-1$ and $t_{W_{A}} \leq 1$ are straightforward. The inequality $t_{W_{A}} \leq t_{M}$, equivalent to $2\left(r_{1}^{2}+r_{2}^{2}-\right.$ $\left.2 r_{1}^{2} r_{2}^{2}\right)+r_{3}^{2}-1 \geq 0$, is a consequence of Lemma $4.3(1)$.

Assume $\cos \frac{\pi}{4} \leq r_{1} \leq \cos \frac{\pi}{8}$.

At the beginning $t=-1$ of the deformation, the trace $\tau$ of $W_{B}$ given by (2.3.3) satisfies

$$
\tau \leq-8 \cos ^{3} \frac{\pi}{4}-12 \cos ^{2} \frac{\pi}{4}+3<-\frac{3}{2} .
$$

By Lemma 2.3.2, this means that $W_{B}$ may become elliptic only after the parameter

$$
t_{B}:=\frac{-\frac{3}{2}+4 \sum r_{i}^{2}-3}{8 r_{1} r_{2} r_{3}}
$$

that corresponds, by (2.3.6), to $x=-\frac{3}{2}$.

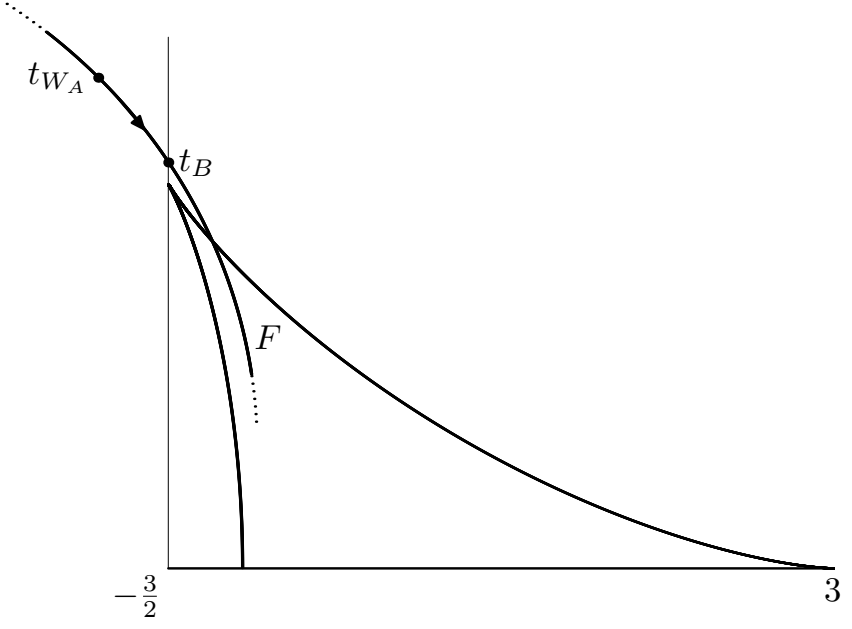


The inequality $t_{W_{A}}<t_{B}$ is equivalent to $8\left(r_{1}^{2}+r_{2}^{2}-2 r_{1}^{2} r_{2}^{2}\right)+4 r_{3}^{2}-5>0$ and follows from Lemma $4.3(2)$. This implies that $\left(n_{1}, n_{2}, n_{3}\right)$ is of type $A$.

We now consider the case $r_{1}=\cos \frac{\pi}{9}$.

By Lemma 2.3.2, intersection points of the deltoid with the line $l:=\left\{(x, y) \in \mathbb{C} \mid y=\frac{3 \sqrt{3}}{5}(1-x)\right\}$ satisfy $(2 x+3)^{2}\left(169 x^{2}-158 x-111\right)=0$. The roots of this equation are $x=-\frac{3}{2}$ (that corresponds to a vertex of the deltoid),

$$
x=x_{1}:=\frac{1}{169}(79-50 \sqrt{10}), \quad \text { and } \quad x=\frac{1}{169}(79+50 \sqrt{10}) .
$$

Intersection points of the circle $F$ given by (2.3.5) with the line $l$ satisfy

$$
52 x^{2}+2\left(25\left(4 \sum r_{i}^{2}-3\right)-27\right) x+25\left(\left(4 \sum r_{i}^{2}-3\right)^{2}-\left(8 r_{1} r_{2} r_{3}\right)^{2}\right)+27=0 .
$$

By Lemma 4.3 (3), the discriminant

$$
D_{2}:=100\left(-27\left(4 \sum r_{i}^{2}-3\right)^{2}-54\left(4 \sum r_{i}^{2}-3\right)+52\left(8 r_{1} r_{2} r_{3}\right)^{2}-27\right)
$$

of the above equation is such that $D_{2}>0$. Take the root

$$
x_{2}:=\frac{-2\left(25\left(4 \sum r_{i}^{2}-3\right)-27\right)-\sqrt{D_{2}}}{104} .
$$

In order to prove that $\left(9, n_{2}, n_{3}\right)$ is of type $A$, it suffices to apply Lemma 2.3 .1 after showing the following facts (see the picture below):

(1) $x_{2}<-\frac{3}{2}$. This implies that, when $F$ crosses $l$ for the first time (thus entering the region in grey), $W_{B}$ has not become elliptic yet.

(2) $x_{W_{A}}<x_{1}$ and $g\left(x_{W_{A}}, y_{W_{A}}\right)>0$, where $\left(x_{W_{A}}, y_{W_{A}}\right) \in F$ is the point that corresponds to $t_{W_{A}}$ by (2.3.6) and $g(x, y):=y-\frac{3 \sqrt{3}}{5}(1-x)$. This implies that we are still in the grey region when $t=t_{W_{A}}$. In particular, $W_{B}$ has not become elliptic yet.

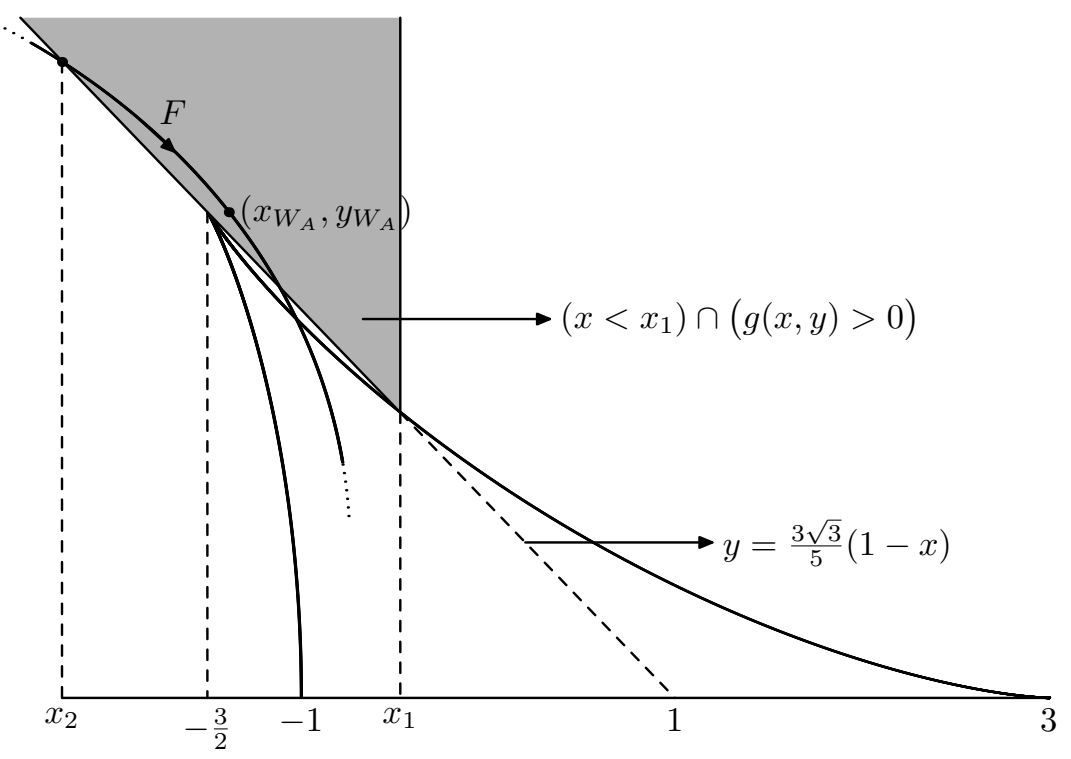


The inequality $x_{2}<-\frac{3}{2}$, equivalent to $\sqrt{D_{2}}>-50\left(4 \sum r_{i}^{2}-3\right)+210$, follows from

$$
-50\left(4 \sum r_{i}^{2}-3\right)+210 \leq-50\left(12 \cos ^{2} \frac{\pi}{9}-3\right)+210 \prec 0 .
$$

By (2.3.6) and (2.3.5),

$$
x_{W_{A}}:=2\left(r_{3}^{2}+4 r_{1}^{2} r_{2}^{2}-1\right)-\left(4 \sum r_{i}^{2}-3\right), \quad y_{W_{A}}:=2 \sqrt{\left(4 r_{1} r_{2} r_{3}\right)^{2}-\left(r_{3}^{2}+4 r_{1}^{2} r_{2}^{2}-1\right)^{2}} .
$$

The inequality $x_{W_{A}}<x_{1}$, equivalent to $2\left(r_{3}^{2}+4 r_{1}^{2} r_{2}^{2}-1\right)-\left(4 \sum r_{i}^{2}-3\right)-\frac{1}{169}(79-50 \sqrt{10})<0$, follows from Lemma 4.3 (4). Finally, $g\left(x_{W_{A}}, y_{W_{A}}\right)>0$ is a consequence of

$$
25\left(\left(4 r_{1} r_{2} r_{3}\right)^{2}-\left(r_{3}^{2}+4 r_{1}^{2} r_{2}^{2}-1\right)^{2}\right)-27\left(2 r_{2}^{2}\left(1-2 r_{1}^{2}\right)+2 r_{1}^{2}+r_{3}^{2}\right)^{2}>0
$$

which follows from Lemma 4.3 (5)

3.6. Remark. For the sake of generality, we have not considered yet the $\left(3, n_{2}, n_{3}\right)$-triangles, where $3 \leq n_{2} \leq n_{3} \leq \infty$. As in Remark 2.2.4, it is easy to see that there exists in general a non-empty one parameter family of $\left(3, n_{2}, n_{3}\right)$-triangles. The only exception is the $(3,3,3)$-triangle, which is rigid. Proceeding as in the first part of the proof of Proposition 3.5 (where we dealt with the $4 \leq n_{1} \leq 8$ cases) and using the fact that $n_{i} \in \mathbb{N}$, one easily shows that the non-rigid $\left(3, n_{1}, n_{2}\right)$-triangles are of type $A$

\section{Taking Derivatives}

4.1. Lemma. Suppose that $\cos \frac{\pi}{14} \leq x, y, z \leq 1$. Define

$$
\begin{aligned}
& f_{1}(x, y, z):=-3\left(4\left(x^{2}+y^{2}+z^{2}\right)-3\right)^{2}+(16 x y z)^{2}, \\
& f_{2}(x, y, z):=-\left(4\left(x^{2}+y^{2}+z^{2}\right)-3\right)^{2}+3\left(4\left(x^{2}+y^{2}+z^{2}\right)-3\right)+(8 x y z)^{2}-9, \\
& f_{3}(x, y, z):=-3\left(4\left(x^{2}+y^{2}+z^{2}\right)-3\right)+32 x y z, \\
& f_{4}(x, y, z):=3\left(4\left(x^{2}+y^{2}+z^{2}\right)-3\right)^{2}-12\left(z^{2}+4 x^{2} y^{2}-1\right)\left(4\left(x^{2}+y^{2}+z^{2}\right)-3\right)+16\left(z^{2}+4 x^{2} y^{2}-1\right)^{2}-(8 x y z)^{2} .
\end{aligned}
$$

Then,

$$
\begin{gathered}
\text { (1) } 31 \prec 4 f_{1}(x, y, z) \leq 52, \quad \text { (2) } 5.5<\sqrt{4 f_{1}(x, y, z)}<7.3, \quad \text { (3) } f_{2}(x, y, z) \succ 0.5, \\
\text { (4) } 2 f_{3}(x, y, z) \succ 8.8, \quad \text { and } \quad \text { (5) } f_{4}(x, y, z) \succ 0.1 .
\end{gathered}
$$

Proof is straightforward. We show, for instance, the first and the last items. Notice that

$$
8 \cos ^{2} \frac{\pi}{14}-3 \succ 0 \text {. }
$$

(1) Taking derivatives,

$$
\frac{\partial f_{1}}{\partial x}=16 x\left(-3\left(4\left(x^{2}+y^{2}+z^{2}\right)-3\right)+32 y^{2} z^{2}\right), \quad \frac{\partial^{2} f_{1}}{\partial x^{2}}=16\left(-36 x^{2}-3\left(4\left(y^{2}+z^{2}\right)-3\right)+32 y^{2} z^{2}\right) .
$$

By (4.2), $\frac{\partial^{2} f_{1}}{\partial x^{2}}$ is increasing in $y$ and in $z$. Hence, $\frac{\partial^{2} f_{1}}{\partial x^{2}} \leq \frac{\partial^{2} f_{1}}{\partial x^{2}}\left(\cos \frac{\pi}{14}, 1,1\right) \prec 0$. This implies that $\frac{\partial f_{1}}{\partial x}$ is decreasing in $x$ and (4.2) implies that it is increasing in $y$ and in $z$. So, $\frac{\partial f_{1}}{\partial x} \geq \frac{\partial f_{1}}{\partial x}\left(1, \cos \frac{\pi}{14}, \cos \frac{\pi}{14}\right) \succ 0$. We have just proved that $f_{1}$ is increasing in every variable. It follows that

$$
31 \prec 4 f_{1}\left(\cos \frac{\pi}{14}, \cos \frac{\pi}{14}, \cos \frac{\pi}{14}\right) \leq 4 f_{1} \leq 4 f_{1}(1,1,1) \leq 52 .
$$


(5) Taking derivatives,

$$
\begin{gathered}
\frac{\partial f_{4}}{\partial x}=16 x\left(4 x^{2}\left(16 y^{4}-12 y^{2}+3\right)-2 y^{2}\left(12 y^{2}+8 z^{2}-7\right)+6 z^{2}-3\right), \\
\frac{\partial^{2} f_{4}}{\partial x \partial y}=64 x y\left(-24\left(x^{2}+y^{2}\right)+64 x^{2} y^{2}-8 z^{2}+7\right), \quad \frac{\partial f_{4}}{\partial z}=8 z\left(12\left(x^{2}+y^{2}\right)-32 x^{2} y^{2}+8 z^{2}-5\right) .
\end{gathered}
$$

Put $g(y):=16 y^{4}-12 y^{2}+3$. Notice that $g^{\prime}(y)>0 \Longleftrightarrow 8 y^{2}-3>0$ and that the last inequality follows from (4.2). Hence, $g(y) \geq g\left(\cos \frac{\pi}{14}\right) \succ 0$. This implies that $\frac{\partial f_{4}}{\partial x}$ is increasing in $x$. By (4.2), $\frac{\partial^{2} f_{4}}{\partial x \partial y}$ is increasing in $x$ and in $y$. Hence, $\frac{\partial^{2} f_{4}}{\partial x \partial y} \geq \frac{\partial^{2} f_{4}}{\partial x \partial y}\left(\cos \frac{\pi}{14}, \cos \frac{\pi}{14}, 1\right) \succ 0$. In other words, $\frac{\partial f_{4}}{\partial x}$ is increasing also in $y$. It is decreasing in $z$ by (4.2). So, $\frac{\partial f_{4}}{\partial x} \geq \frac{\partial f_{4}}{\partial x}\left(\cos \frac{\pi}{14}, \cos \frac{\pi}{14}, 1\right) \succ 0$. It follows that $f_{4}$ is increasing in both $x$ and $y$. Moreover, $\frac{\partial f_{4}}{\partial z}$ is increasing in $z$ and decreasing in $x$ and in $y$ by (4.2). This implies that $\frac{\partial f_{4}}{\partial z} \leq \frac{\partial f_{4}}{\partial z}\left(\cos \frac{\pi}{14}, \cos \frac{\pi}{14}, 1\right) \prec 0$, that is, $f_{4}$ is decreasing in $z$. Finally,

$$
f_{4} \geq f_{4}\left(\cos \frac{\pi}{14}, \cos \frac{\pi}{14}, 1\right) \succ 0.1
$$

\subsection{Lemma. Define}

$g_{1}(x, y, z):=2\left(x^{2}+y^{2}-2 x^{2} y^{2}\right)+z^{2}-1$ for $\cos \frac{\pi}{4} \leq x \leq y \leq z \leq 1$, $g_{2}(x, y, z):=8\left(x^{2}+y^{2}-2 x^{2} y^{2}\right)+4 z^{2}-5$ for $\cos \frac{\pi}{4} \leq x \leq \cos \frac{\pi}{8}$ and $x \leq y \leq z \leq 1$.

Suppose that $x=\cos \frac{\pi}{9}$ and that $\cos \frac{\pi}{9} \leq y \leq z \leq 1$. Define

$$
\begin{aligned}
& g_{3}(y, z):=-27\left(4\left(x^{2}+y^{2}+z^{2}\right)-3\right)^{2}-54\left(4\left(x^{2}+y^{2}+z^{2}\right)-3\right)+52(8 x y z)^{2}-27, \\
& g_{4}(y, z):=2\left(z^{2}+4 x^{2} y^{2}-1\right)-\left(4\left(x^{2}+y^{2}+z^{2}\right)-3\right)-\frac{1}{169}(79-50 \sqrt{10}), \\
& g_{5}(y, z):=25\left((4 x y z)^{2}-\left(z^{2}+4 x^{2} y^{2}-1\right)^{2}\right)-27\left(2 y^{2}\left(1-2 x^{2}\right)+2 x^{2}+z^{2}\right)^{2} .
\end{aligned}
$$

Then,

$$
\text { (1) } g_{1}(x, y, z) \geq 0, \quad \text { (2) } g_{2}(x, y, z) \succ 0.1, \quad \text { (3) } g_{3}(y, z) \succ 296
$$

(4) $g_{4}(y, z) \prec-0.9$, and (5) $g_{5}(y, z) \succ 0.2$.

Proof is straightforward. We show the first and the last items, for instance.

(1) Clearly, $g_{1}(x, y, z) \geq g_{1}(x, y, y)$. Since $g_{1}(x, y, y)=2 x^{2}\left(1-2 y^{2}\right)+3 y^{2}-1$ and $1-2 y^{2} \leq 0$, we obtain $g_{1}(x, y, z) \geq g_{1}(x, y, y) \geq g_{1}(y, y, y)=-4 y^{4}+5 y^{2}-1 \geq 0$.

(5) Taking derivatives,

$$
\frac{\partial g_{5}}{\partial y}=8 y\left(z^{2}\left(104 x^{2}-27\right)+2 y^{2}\left(-100 x^{4}-27\left(1-2 x^{2}\right)^{2}\right)-4 x^{2}\left(1-27 x^{2}\right)\right) .
$$

It follows from $104 x^{2}-27 \succ 0$ and $-100 x^{4}-27\left(1-2 x^{2}\right)^{2}<0$ that $\frac{\partial g_{5}}{\partial y} \leq \frac{\partial g_{5}}{\partial y}\left(\cos \frac{\pi}{9}, 1\right) \prec 0$. In other words, $g_{5}(y, z)$ is decreasing in $y$. Hence, $g_{5}(y, z) \geq g_{5}(z, z)$. Define

$$
h(z):=g_{5}(z, z)=25\left(16 x^{2} z^{4}-\left(z^{2}+4 x^{2} z^{2}-1\right)^{2}\right)-27\left(z^{2}\left(3-4 x^{2}\right)+2 x^{2}\right)^{2} .
$$


We have

$$
\frac{\partial h}{\partial z}=4 z\left(4 z^{2}\left(-208 x^{4}+212 x^{2}-67\right)+25\left(1+4 x^{2}\right)-54 x^{2}\left(3-4 x^{2}\right)\right) .
$$

It follows from $-208 x^{4}+212 x^{2}-67 \prec 0$ that $\frac{\partial h}{\partial z} \leq \frac{\partial h}{\partial z}\left(\cos \frac{\pi}{9}\right) \prec 0$. So, $g_{5}(y, z) \geq h(z) \geq h(1) \succ 0.2$

\section{References}

[Gol] W. M. Goldman, Complex Hyperbolic Geometry, Oxford Mathematical Monographs. Oxford Science Publications. The Clarendon Press, Oxford University Press, New York, 1999

[GoP] W. M. Goldman, J. R. Parker, Complex hyperbolic ideal triangle groups, J. Reine Angew. Math 425 (1992), 71-86

[Pra] A. Pratoussevitch, Traces in complex hyperbolic triangle groups, Geom. Dedicata 111 (2005) $159-185$

[Sch1] R. E. Schwartz, Ideal triangle groups, dented tori, and numerical analysis, Ann. of Math. (2) 153 (2001), 533-598

[Sch2] R. E. Schwartz, Complex Hyperbolic Triangle Groups, Proceedings of the International Congress of Mathematicians, Vol. II, Higher Ed. Press, Beijing (2002) 339-349

[Sch3] R. E. Schwartz, A better proof of the Goldman-Parker conjecture, Geom. Topol. 9 (2005) 1539-1601

[Sch4] R. E. Schwartz, Spherical CR Geometry and Dehn Surgery, Ann. of Math. Stud. 1652007 $(2000)$

[W-G] J. Wyss-Gallifent, Complex Hyperbolic Triangle Groups, Ph. D. thesis, University of Maryland

Departamento de Matemática, ICEX, Universidade Federal de Minas Gerais, 31161-970-Belo Horizonte-MG, Brasil

E-mail address: grossi_ferreira@yahoo.com 